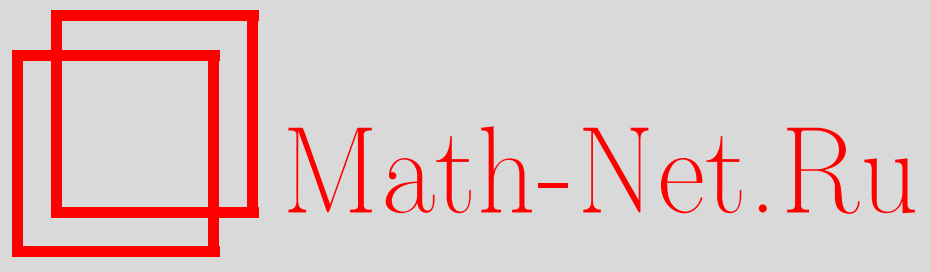

Ю. О. Королева, О неравенстве Фридрихса в трехмерной области, непериодически перфорированной вдоль части границы, УМH, 2010, том 65, выпуск 4, 199-200

DOI: https://doi.org/10.4213/rm9344

Использование Общероссийского математического портала Math-Net.Ru подразумевает, что вы прочитали и согласны с пользовательским соглашением http://www . mathnet.ru/rus/agreement

Параметры загрузки:

IP: 3.85 .5 .30

26 апреля 2023 г., 12:00:42

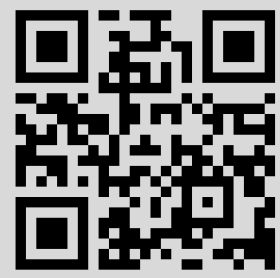




\section{О неравенстве Фридрихса в трехмерной области, непериодически перфорированной вдоль части границы \\ Ю. О. Королева}

В этой работе доказывается трехмерное неравенство типа Фридрихса в перфорированном кубе. Двумерное аналогичное неравенство см. в [1].

Рассмотрим область $\Omega=\{(x, y, z): 0<x<a, 0<y<a, 0<z<a\}$. Пусть $N \gg 1$ - натуральное число, $\varepsilon=a / N$ - малый параметр. Представим $\Omega$ в виде $\Omega=\bigcup_{i, j=1}^{N} \Pi_{\varepsilon}^{i, j}$, где $\Pi_{\varepsilon}^{i, j}=\{(x, y, z) \in \Omega:(i-1) \varepsilon \leqslant x \leqslant i \varepsilon,(j-1) \varepsilon \leqslant y \leqslant j \varepsilon, 0 \leqslant$ $z \leqslant a\}$. Пусть $\varepsilon_{1}^{i, j}=\alpha^{i, j} \varepsilon, 0<\alpha^{i, j}=$ const $<1, \varepsilon_{2}^{i, j}=\beta^{i, j} \varepsilon, 0 \leqslant \beta^{i, j}=$ const, $\max \left\{\alpha^{0,0}+\alpha^{1,0}, \alpha^{0,0}+\alpha^{0,1}\right\} \leqslant \beta^{0,0} \leqslant \mathscr{B}, \max \left\{\alpha^{i, j}+\alpha^{i+1, j}, \alpha^{i, j}+\alpha^{i, j+1}\right\} \leqslant \beta^{i, j} \leqslant \mathscr{B}$, $\mathscr{B}=$ const, $i, j=1, \ldots, N$. Определим $B_{\varepsilon}^{i, j}=\left\{(x, y, z) \in \Pi_{\varepsilon}^{i, j}:\left(x-x_{0}^{i, j}\right)^{2}+\left(y-y_{0}^{i, j}\right)^{2}+\right.$ $\left.(z-\varepsilon)^{2} \leqslant\left(\varepsilon_{1}^{i, j}\right)^{2}\right\}$, здесь $\left|x_{0}^{i+1, j}-x_{0}^{i, j}\right|=\varepsilon_{2}^{i+1, j},\left|y_{0}^{i, j+1}-y_{0}^{i, j}\right|=\varepsilon_{2}^{i, j+1}$ (см. рис.). Пусть

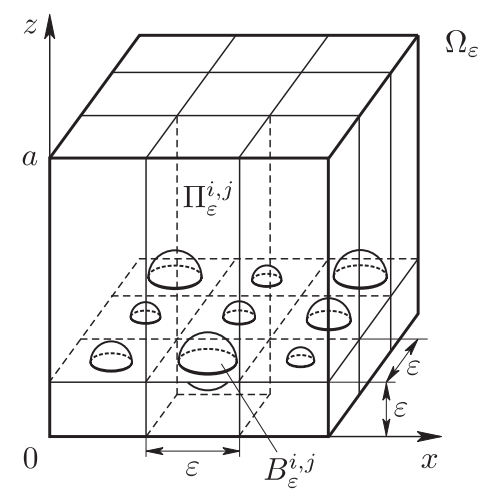

$B_{\varepsilon}=\bigcup_{i, j=1}^{N} B_{\varepsilon}^{i, j}$. Рассмотрим $\Omega_{\varepsilon}=\Omega \backslash \bar{B}_{\varepsilon}, \Gamma_{\varepsilon}=\partial B_{\varepsilon}$. Обозначим $H^{1}\left(\Omega_{\varepsilon}, \Gamma_{\varepsilon}\right)=\{u \in$ $\left.H^{1}\left(\Omega_{\varepsilon}\right):\left.u\right|_{\Gamma_{\varepsilon}}=0\right\}$, аналогично, $C^{\infty}\left(\Omega_{\varepsilon}, \Gamma_{\varepsilon}\right)=\left\{u \in C^{\infty}\left(\Omega_{\varepsilon}\right): u=0\right.$ в окрестности $\left.\Gamma_{\varepsilon}\right\}$. Имеет место следующее утверждение.

Теорема 1. Для $u \in H^{1}\left(\Omega_{\varepsilon}, \Gamma_{\varepsilon}\right)$ справедливо неравенство типа Фридрихса

$$
\int_{\Omega_{\varepsilon}} u^{2} d x d y d z \leqslant C(a) \int_{\Omega_{\varepsilon}}|\nabla u|^{2} d x d y d z, \quad \text { где } C(a) \text { не зависит от } u .
$$

ДоказАтельство. Предположим сначала, что $u \in C^{\infty}\left(\Omega_{\varepsilon}, \Gamma_{\varepsilon}\right)$. Введем обозначения $Q_{\varepsilon}^{i, j}=\left\{(x, y, z) \in \Pi_{\varepsilon}^{i, j}:\left(x-x_{0}^{i j}\right)^{2}+\left(y-y_{0}^{i j}\right)^{2} \leqslant\left(\varepsilon_{1}^{i, j}\right)^{2}, 0 \leqslant z \leqslant a\right\}, \widetilde{\Pi}_{\varepsilon}^{i, j}=$ $\left\{(x, y, z) \in \Pi_{\varepsilon}^{i, j}: \sum_{k=1}^{i} \varepsilon_{2}^{k-1, j}-\varepsilon_{1}^{i, j} \leqslant x \leqslant \sum_{k=1}^{i} \varepsilon_{2}^{k-1, j}+\varepsilon_{1}^{i, j},(j-1) \varepsilon \leqslant y \leqslant j \varepsilon, 0 \leqslant z \leqslant a\right\}$. Далее, пусть $\widetilde{\Pi}^{+; i, j}\left(\widetilde{\Pi}^{-; i, j}\right)$ - подмножество $\widetilde{\Pi}^{i, j}$, ограниченное "спереди" (“сзади”) цилиндром $Q_{\varepsilon}^{i, j}$, и параллелепипед $\widetilde{\Pi}_{\varepsilon}^{i, j ;+}\left(\widetilde{\Pi}_{\varepsilon}^{i, j ;-}\right)$ есть подмножество $\Pi_{\varepsilon}^{i, j}$, ограниченное "слева" ("справа") параллелепипедом $\widetilde{\Pi}_{\varepsilon}^{i, j}$. Кроме того, пусть $Q_{\varepsilon, \omega}^{i, j}=Q_{\varepsilon}^{i, j} \cap\{z \leqslant \omega\}$, $q_{\omega}^{i, j}=Q_{\varepsilon}^{i, j} \cap\{z=\omega\}, \Gamma_{\omega}^{i, j}=\Pi_{\varepsilon}^{i, j} \cap\{z=\omega\}$. Аналогично, $\widetilde{\Gamma}_{\omega}^{i, j}, \widetilde{\Gamma}_{\omega}^{ \pm ; i, j}, \widetilde{\Gamma}_{\omega}^{i, j ; \pm}-$ сечения плоскостью $z=\omega$ областей $\widetilde{\Pi}^{i, j}, \widetilde{\Pi}^{ \pm ; i, j}, \widetilde{\Pi}^{i, j ; \pm}$.

1. Пусть $(x, y, \omega) \in q_{\omega}^{i, j}$, а $\left(x, y, z_{0}\right) \in \partial B_{\varepsilon}^{i, j}$. Следующая цепочка неравенств справедлива в силу формулы Ньютона-Лейбница и неравенства Коши-Буняковского:

$$
u^{2}(x, y, \omega)=\left(\int_{z_{0}}^{\omega} \frac{\partial u}{\partial z} d z\right)^{2} \leqslant \omega \int_{z_{0}}^{\omega}\left(\frac{\partial u}{\partial z}\right)^{2} d z \leqslant \omega \int_{0}^{\omega}|\nabla u|^{2} d z .
$$

Работа выполнена при поддержке РФФИ (грант № 09-01-00530) и программы "Ведущие научные школы" (грант НШ-7429.2010.1). 
Далее, проинтегрируем обе части неравенства (2) по $x, y$ по сечению $q_{\omega}^{i, j}$ а затем еще раз по $\omega$ от 0 до $a$ :

$$
\begin{gathered}
\int_{q_{\omega}^{i, j}} u^{2}(x, y, \omega) d x d y \leqslant \omega \int_{Q_{\varepsilon, \omega}^{i, j}}|\nabla u|^{2} d x d y d z \leqslant a \int_{Q_{\varepsilon}^{i, j}}|\nabla u|^{2} d x d y d z, \\
\int_{Q_{\varepsilon}^{i, j}} u^{2} d x d y d z \leqslant a \omega \int_{Q_{\varepsilon}^{i, j}}|\nabla u|^{2} d x d y d z \leqslant a^{2} \int_{Q_{\varepsilon}^{i, j}}|\nabla u|^{2} d x d y d z .
\end{gathered}
$$

2. Пусть $(x, y, \omega) \in \widetilde{\Gamma}_{\omega}^{ \pm ; i, j}$, а $(\tilde{x}, \tilde{y}, \omega) \in q_{\omega}^{i, j}$, причем $\tilde{x}(x), \tilde{y}(y)$ выбраны так, что $(\tilde{x}, \tilde{y}, \omega) \in q_{\omega}^{i, j}$ при $(x, y, \omega) \in \widetilde{\Gamma}_{\omega}^{ \pm ; i, j}$. Для удобства обозначим $p=(x, y), \tilde{p}=(\tilde{x}, \tilde{y})$. По формуле Ньютона-Лейбница $u(p, \omega)=u(\tilde{p}, \omega)+\int_{\tilde{p}}^{p} \frac{\partial u}{\partial p} d p$. Возведем обе части данного равенства в квадрат и воспользуемся неравенством $(A+B)^{2} \leqslant 2 A^{2}+2 B^{2}$. Учитывая, что $\partial u / \partial p=(\nabla u, \vec{p})$, где $\vec{p}$ - вектор, соединяющий точки $\tilde{p}$ и $p$, и используя неравенство Коши-Буняковского, получаем

$$
\left(\int_{\tilde{p}}^{p} \frac{\partial u}{\partial p} d p\right)^{2} \leqslant C_{1}\left(\varepsilon_{1}^{i, j}, \varepsilon_{2}^{i, j}\right) \int_{\widetilde{\Gamma}_{\omega}^{ \pm ; i, j} \cup q_{\omega}^{i, j}}|\nabla u|^{2} d x d y \leqslant C_{2} \int_{\widetilde{\Gamma}_{\omega}^{ \pm ; i, j} \cup q_{\omega}^{i, j}}|\nabla u|^{2} d x d y,
$$

где константа $C_{2}$ не зависит от $\varepsilon$. Учитывая (4), имеем $u^{2}(x, y, \omega) \leqslant 2 u^{2}(\tilde{x}, \tilde{y}, \omega)+$ $2 C_{2} \int_{\widetilde{\Gamma}_{\omega}^{ \pm ; i, j} \cup q_{\omega}^{i, j}}|\nabla u|^{2} d x d y$. Интегрируя обе части последнего неравенства по $x, y$ по сечению $\widetilde{\Gamma}_{\omega}^{ \pm ; i, j}$, а затем еще раз по $\omega$ от 0 до $a$ и учитывая (3), получаем

$$
\int_{\widetilde{\Pi}_{\varepsilon}^{ \pm ; i, j}} u^{2} d x d y d z \leqslant M^{ \pm}(a) \int_{Q_{\varepsilon}^{i, j} \cup \tilde{\Pi}_{\varepsilon}^{ \pm ; i, j}}|\nabla u|^{2} d x d y d z,
$$

где $M^{ \pm}(a)$ не зависит от $u$.

3. Пусть теперь $(x, y, \omega) \in \widetilde{\Gamma}_{\omega}^{i, j ; \pm}$, а $(\tilde{x}, \tilde{y}, \omega) \in \widetilde{\Gamma}_{\omega}^{i, j}$. При этом $\tilde{x}(x)$ и $\tilde{y}(y)$ выбраны так, что $(\tilde{x}, \tilde{y}, \omega) \in \widetilde{\Gamma}_{\omega}^{i, j ; \pm}$ при $(x, y, \omega) \in \widetilde{\Gamma}_{\omega}^{i, j}$. Аналогично п. 2 можно показать, что

$$
\int_{\widetilde{\Pi}_{\varepsilon}^{i, j ; \pm}} u^{2} d x d y d z \leqslant L^{ \pm}(a) \int_{\widetilde{\Pi}_{\varepsilon}^{i, j} \cup \tilde{\Pi}_{\varepsilon}^{i, j ; \pm}}|\nabla u|^{2} d x d y d z
$$

где константа $L^{ \pm}$не зависит от $u$. Объединяя $(3),(5),(6)$, имеем $\int_{\Pi_{\varepsilon}^{i, j}} u^{2} d x d y d z \leqslant$ $C(a) \int_{\Pi_{\varepsilon}^{i, j}}|\nabla u|^{2} d x d y d z$. Суммируя левую и правую части последнего неравенства по всем $i$ и $j$ от 1 до $N$, получаем (1) для $u \in C^{\infty}\left(\Omega_{\varepsilon}, \Gamma_{\varepsilon}\right)$. Наконец, приближая стандартным образом функции из $H^{1}\left(\Omega_{\varepsilon}, \Gamma_{\varepsilon}\right)$ бесконечно дифференцируемыми функциями (см., например, [2; гл. ІІІ]), получаем справедливость теоремы.

Автор выражает самую искреннюю благодарность своему научному руководителю д. ф.-м. н. Г. А. Чечкину за постановку задачи, внимание и помощь на протяжении работы над статьей.

\section{Список литературы}

[1] G. A. Chechkin, Yu. O. Koroleva, A. Meidell, L.-E. Persson, Russ. J. Math. Phys., 16:1 (2009), 1-16. [2] В. П. Михайлов, Дифференциальные уравнения в частных производных, Наука, М., 1983.

Ю. О. Королева (Yu. О. Koroleva)

Московский государственный университет им. М. В. Ломоносова

E-mail: korolevajula@mail.ru
Представлено В. М. Закалюкиным Принято редколлегией 27.01.2010 